\title{
Comparison of Two Digital Cameras based on Spectral Data Estimation Obtained with Two Methods
}

\author{
Dejana Javoršek*, Tim Jerman, Andrej Javoršek
}

Faculty of Natural Sciences and Engineering, Department of Textiles, Chair of Information and Graphic Arts Technology, University of Ljubljana

Snežniška 5, SI-1000 Ljubljana, Slovenia; dejana.javorsek@ntf.uni-lj.si, tim.jerman@fe.uni-lj.si, andrej.javorsek@ntf.uni-lj.si

Abstract: The aim of our research was to prove that a digital camera does not influence the quality of spectral reflectance estimation and that satisfactory results could be achieved with a low-cost camera instead of using more expensive and complex multispectral devices. For that purpose two digital cameras - Nikon D300 in Nikon D700 were compared by obtaining spectral data from RGB values of a digital camera. For calculation of spectral data two different methods were used - SpecSens method and ImaiBerns method. In the research, two different color charts-ColorChecker DC and ColorChecker SG, were used. Performance of each camera and reflectance estimation approach were evaluated based on $R M S E$ and $\triangle E^{*} a b$. Results showed that in the case of the ImaiBerns method it could be concluded that the obtained reflectance spectra are independent from the used camera, as somewhat slightly better results were obtained with Nikon D700. In the case of SpecSens method, which is based on the determination of the spectral sensitivity of the camera, the choice of the camera had quite an impact on the results. These results are pretty unreliable due to the large color differences $\triangle E^{*} a b$, as this calculation takes into account the standard light (first if you want to calculate XYZ and second the LAB values) and standard colorimetric observer.

Keywords: digital camera Nikon D300; digital camera Nikon D700; spectral reflectance estimation

\section{Introduction}

Digital cameras have become very accurate systems for identifying changes in color, for example on beef [1], in the field of phenology [2, 3], in pattern recognition [4], for the identification of colors in urban environments [5], in the field of culture, where the digitization or digital archiving is used for the needs of various cultural institutions [6] or in medicine for photography of human wounds [7]. 
The sensitivities of digital camera differ from CIE color matching functions, which describe the sensitivity of the human visual system. Digital camera could provide two metamerically identical images while human observers could see those images differently [8]. It is known that a color match for all observers when changing illumination could be achieved only by matching spectral data that are completely independent of the characteristics of digital camera. Obtaining spectral data from digital camera RGB values could provide a new way of using digital camera as spectrophotometric tool, where spectral data enables later reproduction under different illuminants and observing conditions. That could be very important and useful for digital archives, network museums, e-commerce and telemedicine [9]. Even after almost 25 years since Glassner wrote about deriving a spectrum from an RGB values [10], this is still a very hot topic. Today, one method to solve this problem is to use a regular digital RGB camera and estimate Suitableits RGB image into a spectral image by the Wiener estimation method [11]. This method was also used for spectral reflectance images obtained from a digital RGB image for estimation of melanin concentration, blood concentration, and oxygen saturation in human skin tissue [12].

Adequate results of obtained spectral data could be also achieved by combining two different shots of the same scene acquired using the digital RGB camera with and without a properly chosen absorption filter [13].

There have been a number of studies on determining camera spectral sensitivity. For defining camera spectral sensitivity, a monochromator or narrow-band filters for generating a series of monochromatic light, are usually used. Other methods that do not use a monochromator require both input images and corresponding scene spectral radiances [14-19]. Thomson and Westland introduced a novel method to estimate camera spectral sensitivities and white balance setting from images with sky regions [20]. In our research instead of a monochromator, a less expensive and more readily available tools - diffraction grating and spectroradiometer were used to determine spectral sensitivities of two commercial digital cameras [21].

In one study authors researched the influence of camera parameters, e.g. exposure, on spectral data reconstruction of prints [22]. They found out that with multiple exposures it is possible to capture high dynamic range images, because limited dynamic range is the factor that lowers the reconstruction performance of consumer level cameras.

The aim of our research was to prove that the digital camera does not or only slightly influences the quality of spectral reflectance estimation and that satisfying results could be achieved with a low-cost camera instead of using more expensive and complex multispectral devices. For that purpose two digital cameras - Nikon D300 and Nikon D700 were compared while obtaining spectral data from RGB values of a digital camera. For calculation of spectral data two different methods were used. The first method was performed using spectral responses of a digital 
camera (SpecSens method), and second one was Imai and Berns (ImaiBerns) method that includes linearized RGB values [23]. In the research, two different color charts - ColorChecker DC and ColorChecker SG, were used. ColorChecker DC was used only in case of the ImaiBerns method, as training set data and for the linearization method. Performance of each camera and reflectance estimation approach were evaluated based on root-mean-square-error (RMSE) and color difference equation $\Delta \mathrm{E}^{*} \mathrm{ab}$.

\section{Experimental}

\subsection{Materials and Methods}

Color charts ColorChecker DC (X-Rite) - training set with 237 color patches and ColorChecker SG (X-Rite) - test set with 140 patches, were photographed with two digital cameras - Nikon D300 in Nikon D700. Charts were illuminated by two light sources with color temperature $3194 \mathrm{~K}$ at $45^{\circ}$ angle and distance of $170 \mathrm{~cm}$. Camera settings were as follows - aperture: f/1.4, ISO value: 200, metering mode: Matrix, captured image format: RAW. Raw images were converted to TIFF files using an open source program dcraw [24].

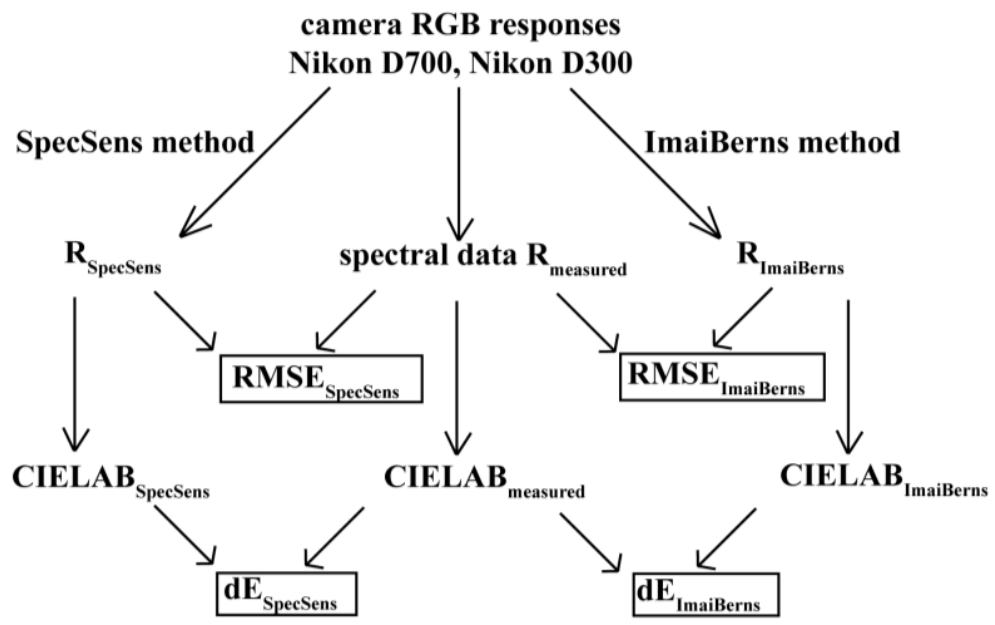

Figure 1

Workflow of the spectral reflectance estimation study, $\mathrm{dE}=\Delta \mathrm{E}^{*} \mathrm{ab}$

Further characterisation steps, i.e. conversion from the RGB responses to the spectral reflectance data and other workflow details are depicted in Figure 1. Spectral reflectance estimation was carried out using two different approaches described below. The obtained results (denoted RSpecSens and RImaiBerns) were compared to the actual reflectance values acquired by the spectrophotometer 
EyeOne (denoted Rmeasured). Performance of the two reflectance estimation procedures was assessed using two error metrics - color difference formula CIE $\triangle \mathrm{E}^{*} \mathrm{ab}$ and RMSE. For calculation of CIE $\triangle \mathrm{E}^{*} \mathrm{ab}$ and CIELAB values illuminant $A$ and CIE 1931 standard observer were used. RMSE is a spectral measure of estimation quality and compares measured and estimated reflectance values on image pixel location in each patch on a pixel-by-pixel basis.

\subsection{Spectral Reflectance Estimation}

The inverse problem of estimating spectral reflectances from the RGB values is related to the image acquisition process that describes the creation of camera responses. The process is described in [25]:

$\mathbf{P}=\mathbf{f}(\Delta \lambda, \mathbf{Y}, \mathbf{l}, \mathbf{R}, \mathbf{b})=\Delta \lambda * \mathbf{Y}^{\prime} \times \operatorname{diag}(\mathbf{l}) \times \mathbf{R}+\mathbf{b}$

where $\Delta \lambda$ denotes the sampling interval of the spectral data, $\mathrm{Y}$ are the spectral responsitivities of dimension $\mathrm{w} \times \mathrm{c}, 1$ is the illumination vector of dimension $\mathrm{w}, \mathrm{R}$ is the reflectance matrix consisting of the $\mathrm{n}$ object pixels in the image and $\mathrm{b}$ is an additive noise term. In spectral reflectance estimation one attempts to calculate unknown reflectances $\mathrm{R}$ from known camera responses $\mathrm{P}$ by finding a function $\mathrm{d}$ that minimizes $d(g(P), R)$. Here $d$ is an error metrics, such as RMSE. Since dimensionality $\mathrm{w}$ of $\mathrm{R}$ is typically larger than $\mathrm{c}$ of $\mathrm{P}, \mathrm{g}$ does not necessarily have a unique solution [26].

\subsubsection{Spectral Sensitivity-based Method (SpecSens)}

For the purpose of our study, the Octave [27] function 'xyz2r' described in [23] that is used to estimate reflectance spectrum from tristimulus values was modified. Values for CIEXYZ and the standard illuminant/observer combination that are required as the function inputs were replaced by camera RGB responses and its sensors' spectral sensitivities.

In our research, to determine camera spectral sensitivities, a diffraction grating and a spectroradiometer were used instead of a monochromator [21]. A transmissive diffraction grating with 590 slits per $\mathrm{mm}$ was placed on the focal point of a biconvex lens onto which light from the illuminant A was projected. The diffraction grating split the parallel rays into several beams travelling in different directions depending on the wavelength. The resulting rainbow was photographed by the Nikon D300 and the Nikon D700 (Figure 2) and measured at several points by means of a spectroradiometer PR650 (X-Rite). From the obtained RAW data, the RGB values of the rainbow were read and a calculation of the corresponding wavelengths was performed. The interpolation of those points produced the spectral response curves for the camera. Since these curves still contained information about the input light spectrum, we divided the values by the interpolated measured intensity values from the spectroradiometer. Finally, the RGB curves were normalized so that the areas under the three curves were equal (Figure 3). 


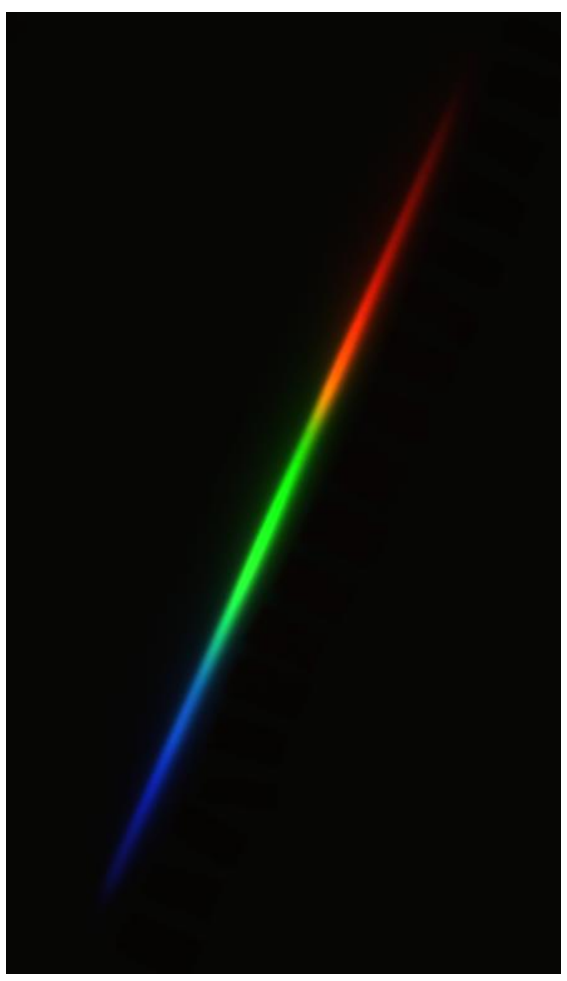

a)

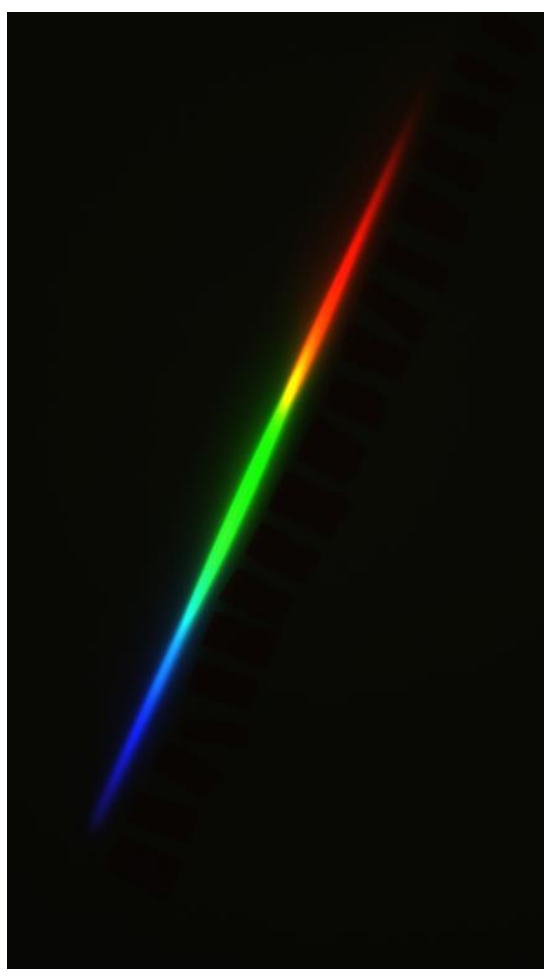

b)

Figure 2

Rainbow photographed by a) Nikon D300 and b) Nikon D700

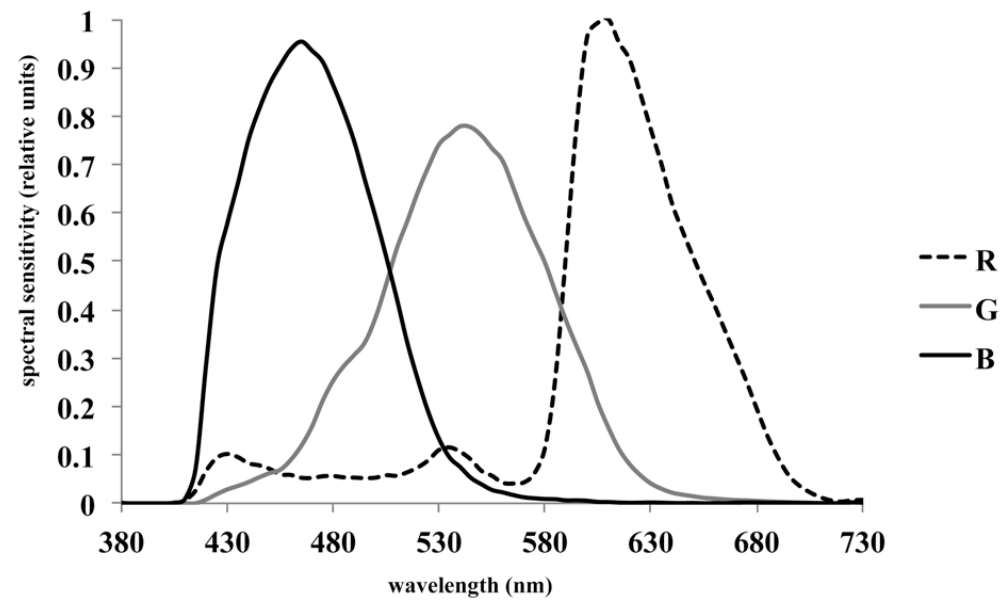

a) 


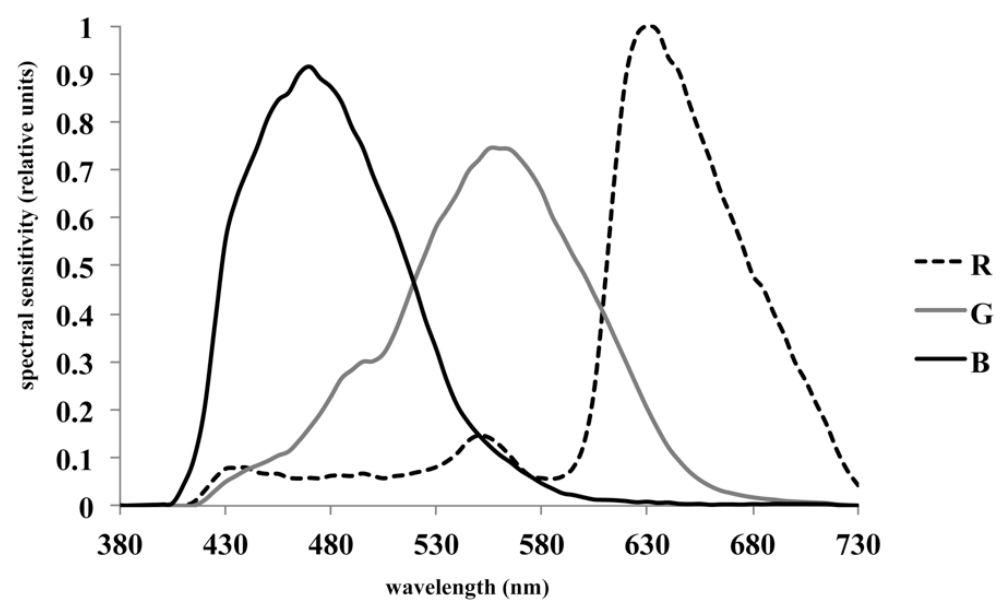

b)

Figure 3

Spectral sensitivity curves of the camera sensors, a) Nikon D700 and b) Nikon D300

\subsubsection{Imai-Berns Method (ImaiBerns)}

First, linearization of the camera RGB responses was performed in order to compensate for the non-linearity introduced by the cameras manufacturer. The RGB values for 12 grey patches of the ColorChecker SG target and the corresponding CIE Y values representing lightness were used for this purpose (Figure 4). CIE Y values were calculated according to ISO 14524 [28]. Based on these data, linearization of all the 140 RGB values was accomplished using Octave functions 'getlincam' and 'lincam' [23].

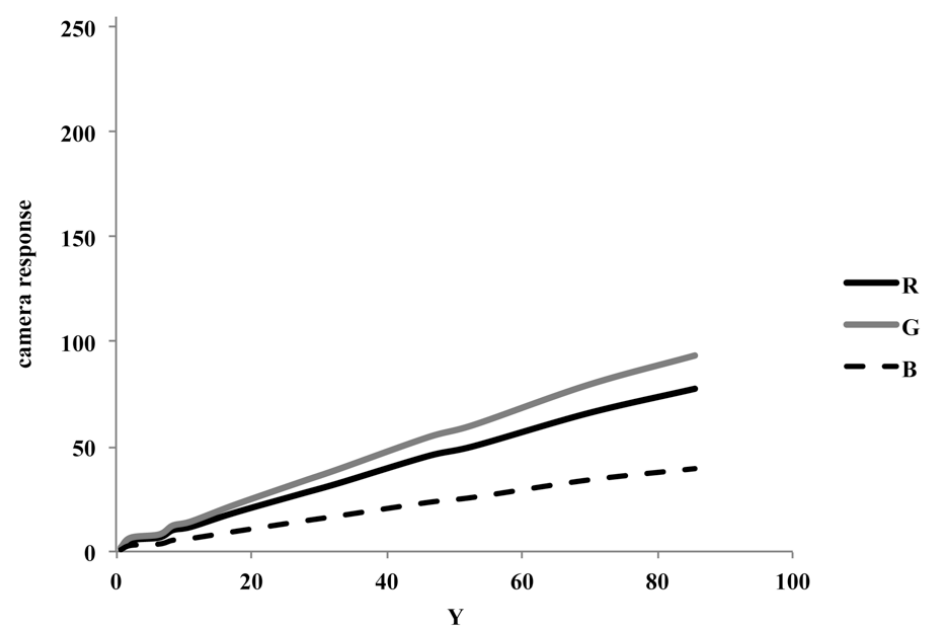

a) 


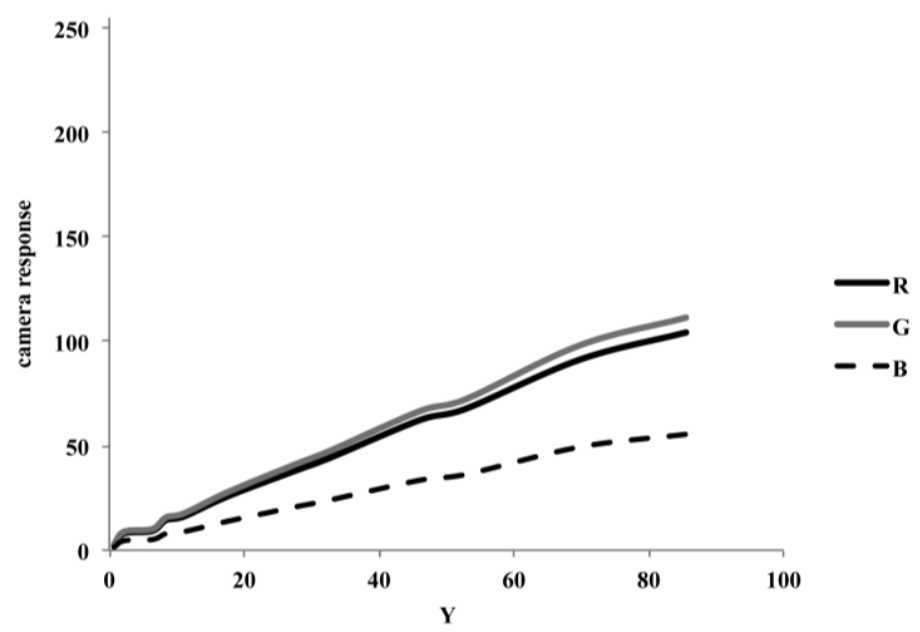

b)

Figure 4

Linearization of the camera RGB responses, a) Nikon D700 and b) Nikon D300

Next, the linearized RGB responses were processed to obtain reflectance estimates using ImaiBerns approach $[8,21]$. The method is based on running principal component analysis (PCA) on a training dataset. $\mathbf{R}_{t r}$ is expressed as a linear combination of $k$ eigenvectors in a $w \times k$ matrix $\mathbf{V}$. The $n_{t r}$ training reflectances are projected to the eigenvectors producing a $k \times n_{t r}$ coefficient matrix $\mathbf{E}_{t r}=(\mathbf{V})^{T}$. $\mathbf{P}_{t r}$ is then related to the coefficient matrix $\mathbf{E}_{t r}$ as $\mathbf{G}=\mathbf{E}_{t r}\left(\mathbf{P}_{t r}\right)^{+}$using least squares regression. Superscript ${ }^{+}$denotes the pseudoinversion of the $\mathbf{P}_{t r}$ matrix. Estimation of unknown reflectances from camera responses of the test set $\mathbf{P}_{t e}$ is calculated as $\mathbf{R}_{t r}=\mathbf{V G P}_{t e}$. The parameter $k$ is obtained by exhaustive search with the goal to minimize the RMSE between the measured and the estimated reflectances in the training set of samples $\mathrm{S}_{t r}$.

The same scene and illuminant were used for both digital cameras so mutual comparison could be achieved.

\section{Results and Discussion}

Results for two methods used for reflectance estimation in terms of two error metrics, $\Delta \mathrm{E}^{*}$ ab and RMSE, are represented in Table 1.

Comparison of both cameras using the colorimetric performance measure $\left(\Delta \mathrm{E}^{*} \mathrm{ab}\right)$ showed better results for the Nikon D700 camera, but according to large values of $\Delta \mathrm{E}^{*} \mathrm{ab}$ it is difficult to confirm that those results are reliable. In the case of the spectral metric RMSE, only the ImaiBerns method showed slightly better results 
for Nikon D700 compared to Nikon D300. The reason for this could be found in the 12-megapixel FX (full-frame) sensor of the Nikon D700, which means it has a more dynamic range and higher ISO, while the Nikon D300 has a smaller 12megapixel DX (1.5 crop factor) sensor.

Table 1

Performance of the methods used for reflectance estimation

\begin{tabular}{|c|c|c|c|c|c|c|c|c|}
\hline & $\Delta \mathbf{E}^{*}{ }_{\text {ab }}$ & $\begin{array}{l}\Delta \mathbf{E}_{\text {ab }}^{*} \\
\text { ImaiBer }\end{array}$ & RMSE & $\begin{array}{l}\text { RMSE } \\
\text { ImaiBer }\end{array}$ & & & & \\
\hline & SpecSens & & SpecSens & & & & & \\
\hline & D700 & D300 & D700 & D300 & D700 & D300 & D700 & D300 \\
\hline mean & 12.87 & 17.39 & 6.32 & 6.95 & 0.1332 & 0.1026 & 0.0596 & 0.0668 \\
\hline $\max$ & 35.04 & 113.17 & 27.37 & 27.78 & 0.3856 & 0.2898 & 0.2150 & 0.2723 \\
\hline $\min$ & 1.59 & 0.72 & 0.47 & 0.39 & 0.0132 & 0.0116 & 0.0015 & 0.0017 \\
\hline
\end{tabular}

Both color difference calculations, $\Delta \mathrm{E}^{*} \mathrm{ab}$ as well as spectral metric RMSE, clearly indicate that the reflectance estimation based on the linearized ImaiBerns method performs better compared to the alternative method (SpecSens) using defined camera spectral sensitivities. It should be noted that the differences between the two methods are almost the same when using both evaluation methods (RMSE and $\Delta \mathrm{E}^{*} \mathrm{ab}$ ): for example, in the case of $\Delta \mathrm{E}^{*} \mathrm{ab}$ the corresponding ratio of mean values SpecSens/ImaiBerns is $2.04(=12.87 / 6.32)$ compared to 2.23 in case of the RMSE.

In order to get a more detailed picture about which color patches can be estimated more or less accurately in terms of their reflectance values, the following results are presented. Colors with the lowest $\Delta \mathrm{E}^{*}$ ab are colors with a mostly high $\mathrm{L}^{*}$ value. In order to get a more detailed information about color shift of these colors when their reflectance spectra were estimated from RGB values, they were represented in $a^{*}, b^{*}$ diagram (Figures 5 and 6).

Color difference $\left(\Delta \mathrm{E}^{*} \mathrm{ab}\right)$ between measured and predicted $\mathrm{L}^{*} \mathrm{a} \mathrm{b}^{*}$ values using ImaiBerns method is the most pronounced in the case of black (patches L01, N06, N03, J05), saturated red (G04, M02, L03, L06, M05), blue (J04) and violet (M03) patch samples (Figure 5). Color difference $\left(\Delta \mathrm{E}^{*} \mathrm{ab}\right)$ between measured and predicted $\mathrm{L}^{*} \mathrm{a} \mathrm{b}^{*}$ values using SpecSens method is the most pronounced in case of black (patches I01, L01, N06, N03), saturated red (G04, M02, L03) and green (L07, L08, L09, G09, I09, H09) patch samples (Figure 6). Lightness of colors captured with Nikon D300 is slightly higher in comparison with the Nikon D700. Lightness of colors captured with the Nikon D700 and obtained with SpecSens method is slightly higher than in the case of the Nikon D300, which is quite opposite of the ImaiBerns method. However, as mentioned above, according to large values of $\Delta \mathrm{E}^{*} \mathrm{ab}$ it is difficult to confirm that results of the SpecSens method are reliable. 


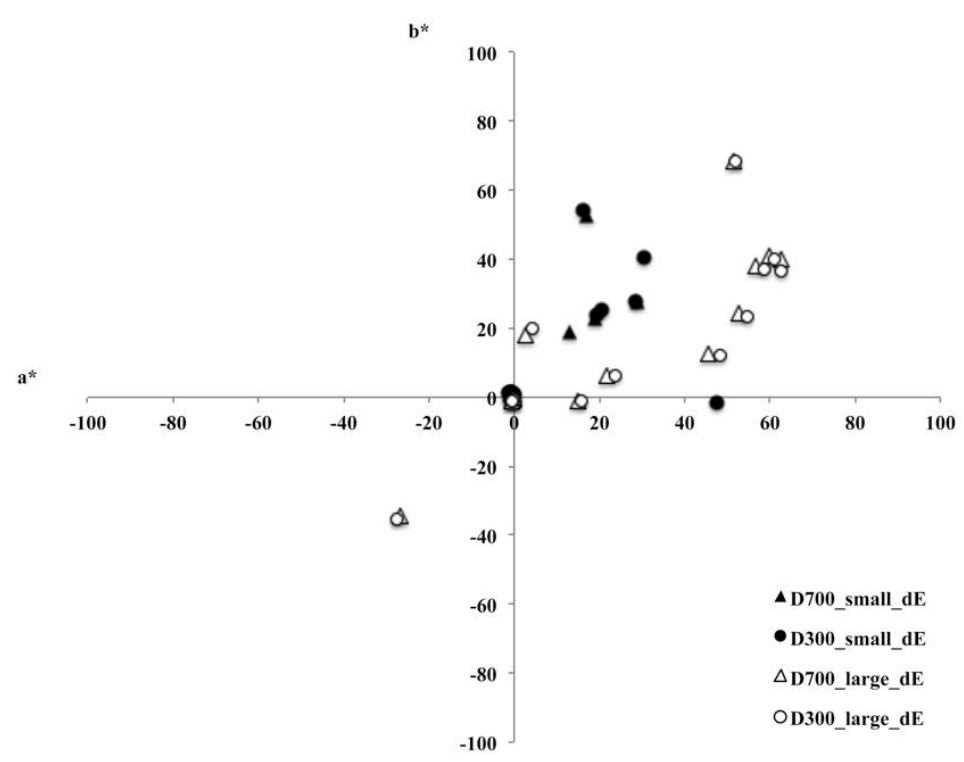

Figure 5

15 colors with the lowest and 15 colors with the highest $\Delta \mathrm{E}^{*} \mathrm{ab}$, obtained with the ImaiBerns method, represented in $a^{*}, b^{*}$ diagram

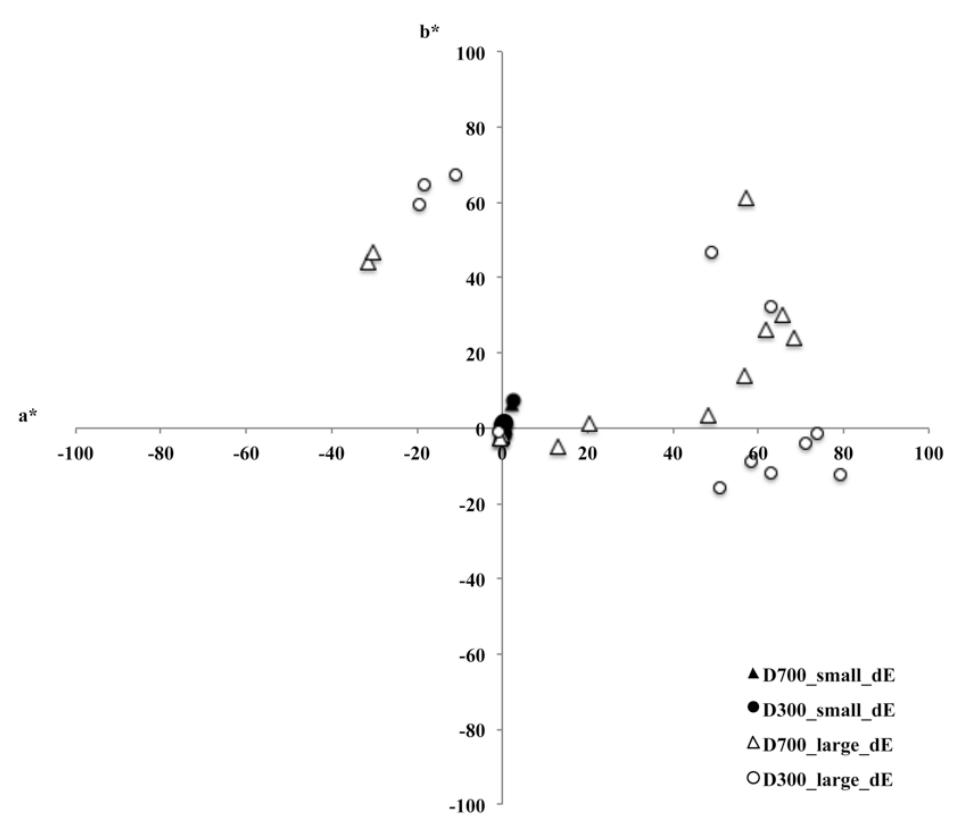

Figure 6

15 colors with the lowest and 15 colors with the highest $\Delta \mathrm{E}^{*} \mathrm{ab}$, obtained with the SpecSens method, represented in $a^{*}, b^{*}$ diagram 
On the other hand, it is also interesting to investigate patch samples with the lowest values of the $\Delta \mathrm{E}^{*} \mathrm{ab}$. Not surprisingly, patches with the lowest $\Delta \mathrm{E}^{*} \mathrm{ab}$ are almost exclusively white, gray and skin color shade samples (ImaiBerns method).

Based on Figures 5 and 6 it can be concluded that in the case of the ImaiBerns method, the Nikon D700 colors with large $\Delta \mathrm{E}^{*} \mathrm{ab}$ generally moved toward the $\mathrm{b}^{*}$ axis, meaning that they are less saturated than in the case of D300. In the case of the SpecSens method, large $\Delta \mathrm{E}^{*} \mathrm{ab}$ colors of the digital camera D700 moved toward the upper half of the $a^{*}, b^{*}$ diagram - their $b^{*}$ values are positive, which means that colors moved from magenta area to red area of the diagram. In a case of yellow, colors moved from yellow area to green area of the diagram.

Figures 7-10 show the obtained spectral data of colors with the maximum and minimum $\Delta \mathrm{E}^{*} \mathrm{ab}$ between the measured and the predicted $\mathrm{L}^{*} \mathrm{a}{ }^{*} \mathrm{~b}^{*}$ values.

The spectral data represented in graphs confirmed previous findings that the difference between the cameras is small, except in case when SpecSens method was used, which unfortunately proved to be the less reliable method for comparing these types of cameras. From Figures 7 and 8 it is more clearly evident that small difference between the cameras is obtained in case of bright color (in Figure 7 white patch A04).

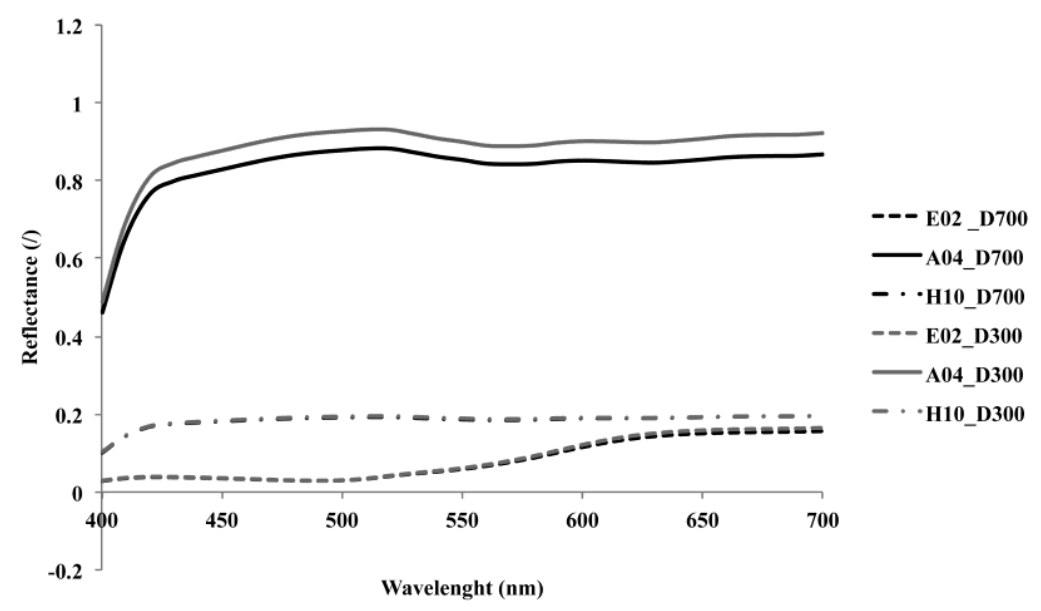

Figure 7

Reflectance spectra for six color patches obtained with the ImaiBerns method with small $\Delta \mathrm{E}^{*} \mathrm{ab}$ 


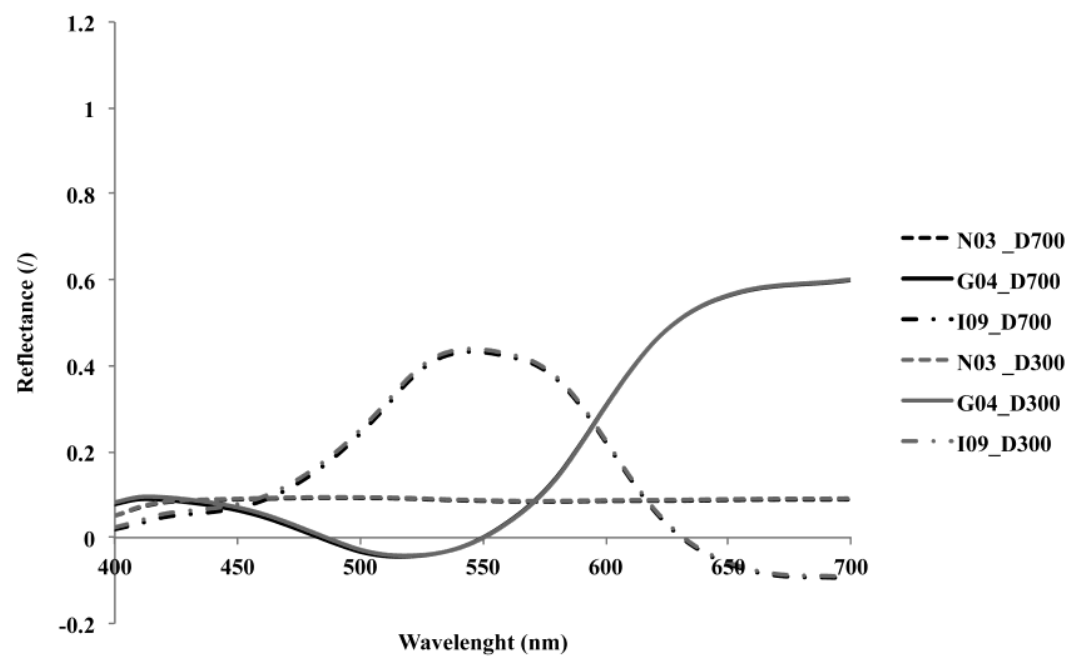

Figure 8

Reflectance spectra for six color patches obtained with the ImaiBerns method with large $\Delta \mathrm{E}^{*} \mathrm{ab}$

On the basis of spectral data in case of SpecSens method it could be seen that there is a difference between the cameras in both bright and dark colors, since for each camera separately spectral sensitivity was measured and calculated. This could lead to significant errors.

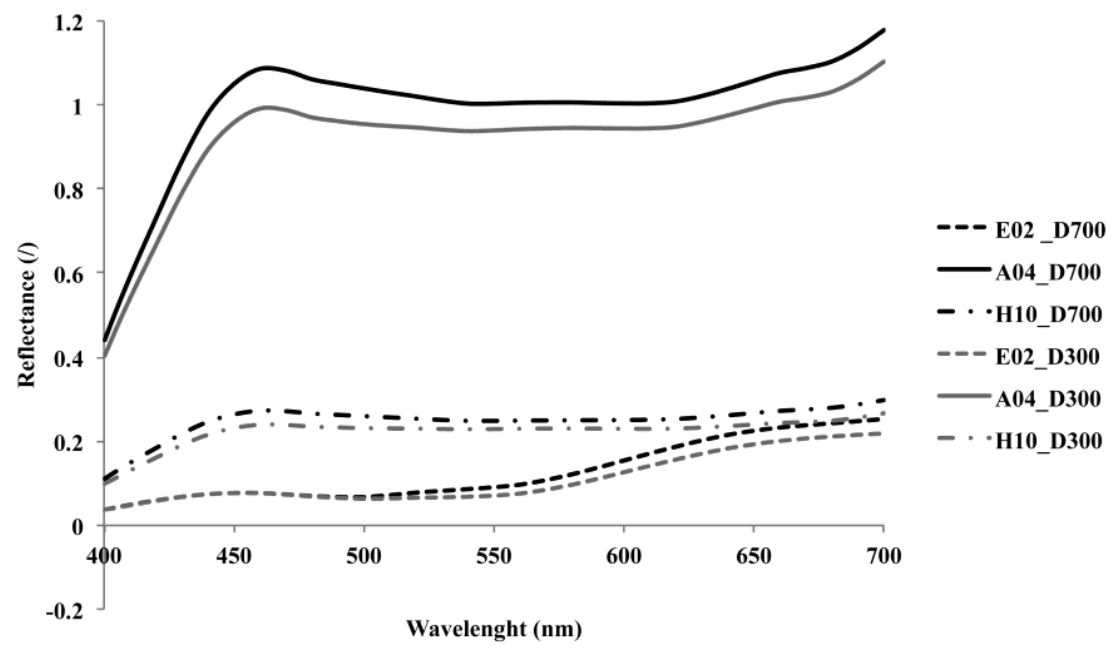

Figure 9

Reflectance spectra for six color patches obtained with the SpecSens method with small $\Delta \mathrm{E}^{*} \mathrm{ab}$ 


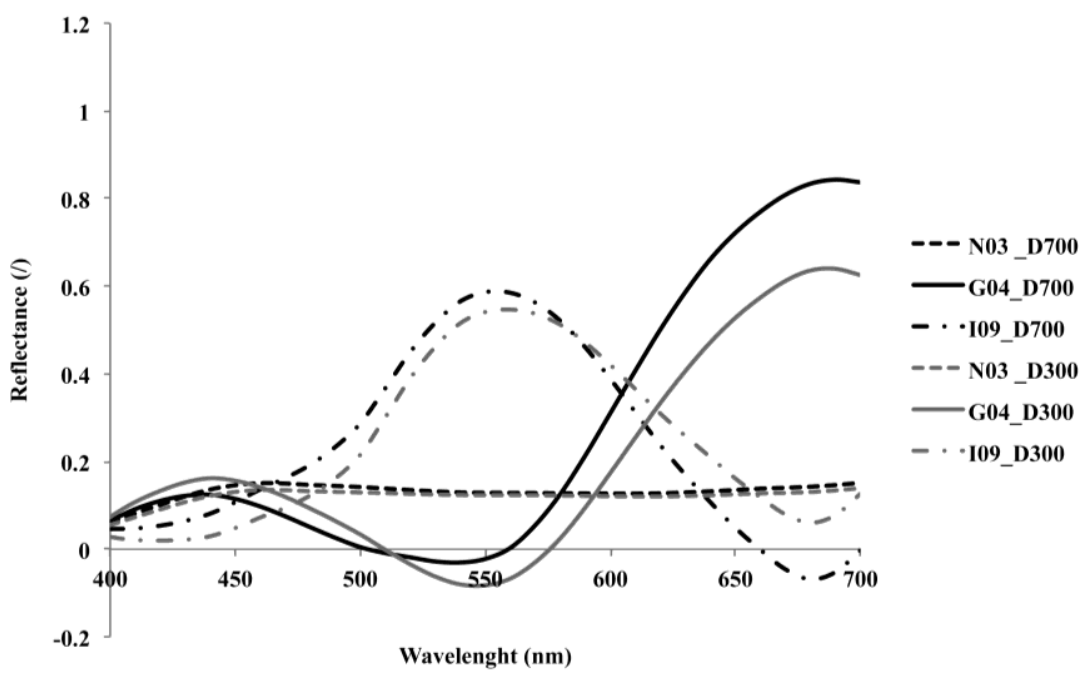

Figure 10

Reflectance spectra for six color patches obtained with the SpecSens method with large $\Delta \mathrm{E}^{*} \mathrm{ab}$

\section{Conclusion}

In our study a comparison of two digital cameras Nikon D300 and Nikon D700 based on spectral data estimation obtained with two methods (ImaiBerns and SpecSens), was performed. Results showed that in the case of using SpecSens method and $\Delta E^{*}$ ab calculations, Nikon D700 had better results than Nikon D300. These results are pretty unreliable due to the large color differences $\Delta \mathrm{E}^{*} \mathrm{ab}$, as this calculation takes into account the standard light (firstly when calculating XYZ and secondly when calculating LAB values) and standard colorimetric observer. In a case of SpecSens method measurement and calculation errors probably also occurred. In the case of the spectral metric RMSE, only the usage of the ImaiBerns method showed negligibly better results for Nikon D700 in comparison with Nikon D300.

Both evaluation methods, $\Delta \mathrm{E}^{*} \mathrm{ab}$ as well as RMSE, clearly indicate that the reflectance estimation, based on the linearized ImaiBerns method, performs better compared to the SpecSens method using defined camera spectral sensitivities. The reason for this could be found in the potential errors that occurred either in the actual measurements (SpecSens method) or calculations.

In the case of the ImaiBerns method it could be concluded that the obtained reflectance spectra are independent from the used camera, as somewhat negligibly better results were obtained with the Nikon D700.

According to $\Delta \mathrm{E}^{*}$ ab calculations between measured and predicted $\mathrm{L}^{*} \mathrm{a}^{*} \mathrm{~b}^{*}$ values ImaiBerns method was the most pronounced in case of black, saturated red and blue patch samples, while SpecSens method was the most pronounced in case of 
black, saturated red and green patch samples. Patches with the lowest $\Delta \mathrm{E}^{*} \mathrm{ab}$ are almost exclusively white, grey and also skin color shade samples in the case of ImaiBerns method.

\section{References}

[1] R. E. Larraín, D. M. Schaefer, J. D. Reed, "Use of Digital Images to Estimate CIE Colour Coordinates of Beef”, Food Research International, Vol. 41, pp. 380-385, 2008

[2] R. Ide, H. Oguma, "Use of Digital Cameras for Phenological Observations”, Ecological Informatics, Vol. 5, No. 5, pp. 339-347, 2010

[3] N. A. Clark, R. H. Wynee, D. L. Schmoldt, M. Winn, "An Assessment of the Utility of a Non-Metric Digital Camera for Measuring Standing Trees", Computer and Electronics in Agriculture, 28, No. 2, pp. 151-169, 2000

[4] M. Tsutida, K. Yano, K. Hachimura, S. Tanaka, K. Furukawa, T. Nishiura, W. Choi, W. Wakit, H. T. Tanaka, "Development of a High-Definition and Multispectral Image Capturing System for Digital Archiving of Early Modern Tapestries of Kyoto Gion Festival", Pattern Recognition (ICPR), $20^{\text {th }}$ International Conference, Istanbul, Turkey, 2010

[5] M. Starešinič, B. Simončič, S. Bračko, "Using a Digital Camera to Identify Colors in Urban Environments", Journal of Imaging Science and Technology, Vol. 55, No. 6, pp. 1-4, 2011

[6] F. B. Wheeler, M. J. Bennett, "Accurate Color: a Preliminary Investigation into the Colour Gamut of Selected Special Collection Library Objects", UConn Libraries Published Works, accessed August 2011, available from http://digitalcommons.uconn.edu/libr_pubs/37

[7] S. Van Poucke, "Automatic Colorimetric Calibration of Human Wounds", accessed June 2014, available from http://www.biomedcentral.com/1471$2342 / 10 / 7$

[8] F. H. Imai and R. S. Berns, "Spectral Estimation using Trichromatic Digital Cameras", in Proc. of the International Symposium on Multispectral Imaging and Color Reproduction for Digital Archives, Chiba University, Chiba, Japan, pp. 42-49, 1999

[9] Y. Miyake, "Evaluation of Image Quality Based on Human Visual Characteristics", Proc. of the First International Workshop on Image Media Quality and its Applications, Nagoya, Japan, pp. 10-14, 2005

[10] A. S. Glassner, "How to Derive a Spectrum from an RGB Triplet", Computer Graphics and Applications, IEEE, Vol. 9, No. 4, pp. 95-99, 1989

[11] P. Stigell, K. Miyata, M. Hauta-Kasari, "Wiener Estimation Method in Estimating of Spectral Reflectance from RGB Images", Pattern Recognition and Image Analysis, Vol. 17, No. 2, pp. 233-242, 2007 
[12] I. Nishidate, T. Maeda, K. Niizeki, Y. Aizu, "Estimation of Melanin and Hemoglobin Using Spectral Reflectance Images Reconstructed from a Digital RGB Image by the Wiener Estimation Method", Sensors, Vol. 13, pp. 7902-7915, 2013

[13] S. Bianco, F. Gasparini, R. Schettini, "Spectral-based Color Imaging using RGB Digital Still Cameras: Simulated Experiments", accessed June 2014, available from http://www.ivl.disco.unimib.it/papers2003/articolo_dsc_multispettrale.pdf

[14] R. Slavuj, P. Green, "To Develop a Method of Estimating Spectral Reflectance from Camera RGB Values", Colour and Visual Computing Symposium, accessed June 2014, available from http://www.google.si/url?sa=t\&rct=j\&q=\&esrc=s\&source=web\&cd=34\&ve $\mathrm{d}=0 \mathrm{CDsQFjADOB} 4 \&$ url=http $\% 3 \mathrm{~A} \% 2 \mathrm{~F} \% 2 \mathrm{Fcolorlab} . n \% \% 2 \mathrm{Fcontent} \% 2 \mathrm{Fdo}$ wnload\%2F42497\%2F569619\%2Ffile\%2FCVCS-

Radovan2013.pdf\&ei $=$ s6c1U-

wDYeCzAPb8oHYDQ\&usg=AFQjCNFbUTd62J68gtXT-

9JSfwl23PB0Xg\&bvm=bv.63808443,d.bGE

[15] P. M. Hubel, D. Sherman, J. E. Farrell, "A Comparison of Method of Sensor Spectral Sensitivity Estimation", Proceedings of Color Science, System, and Application, pp. 45-48, 1994

[16] G. Sharma, H. J. Trussell, "Characterization of Scanner Sensitivity", Proceedings of Transforms and Transportability of Color, pp. 103-107, 1993

[17] G. Finlayson, S Hordley, P Hubel, "Recovering Device Sensitivities with Quadratic Programming", Proceedings of Color Science, System, and Application, pp. 90-95, 1998

[18] K. Barnard, B. Funt, "Camera Characterization for Color Research", Color Research and Application, Vol. 27, No. 3, pp. 153-164, 2002

[19] M. Ebner, "Estimating the Spectral Sensitivity of a Digital Sensor using Calibration Targets", Proceedings of Conference on Genetic and Evolutionary Computation, pp. 642-649, 2007

[20] R. Kawakami, H. Zhao, R. T. Tan, K. Ikeuchi, "Camera Spectral Sensitivity and White Balance Estimation from Sky Images", International Journal of Computer Vision, Vol. 105, No. 3, pp. 187-204, 2013

[21] D. Javoršek, T. Jerman, B. Rat, A. Hladnik, "Assessing the Performance of a Spectral Reflectance Estimation Method based on a Diffraction Grating and a Spectroradiometer", Coloration Technology, Vol. 130, pp. 288-295, 2014

[22] M. Nuutinen, P. Oittinen, "Recovering Spectral Data from Digital Prints with an RGB Camera using Multi-Exposure Method", ISI\&T's Fourth 
European Conference on Colour in Graphics, Imaging and Visualization 2010, pp. 120-125, 2010

[23] S. Westland, C. Ripamonti, "Computational Color Science using MATLAB”, Chichester: John Wiley \& Sons, p. 181, 131, 134, 2004

[24] "Decoding Raw Digital Photos in Linux", accessed July 2014, available from http://www.cybercom.net/ dcoffin/dcraw/

[25] T. Eckhard, E. M. Valero, J. Hernández-Andrés, “A Comparative Analysis of Spectral Estimation Approaches Applied to Print Inspection", 18 Workshop of the German Color Group, Darmstadt, Germany, pp. 13-24, 2012

[26] A. Ribes, F. Schmitt, "Linear Inverse Problems in Imaging", Signal Processing Magazine, IEEE, Vol. 25, No. 4, pp. 84-99, 2008

[27] "GNU Octave", accessed July 2014 available from http://www.gnu.org/software/octave/; last accessed 8 July 2014

[28] Photography - Electronic still-picture cameras - Methods for measuring opto-eletronic conversion functions (OECFs) SIST ISO 14524:2011, Basel: ISO, p. 23, 179-185, 2011 